OPEN ACCESS

Edited by:

Kathrin Jansen,

Pfizer Inc, United States

Reviewed by:

Joanna Kirman,

University of Otago, New Zealand

Zhou Xing,

McMaster University, Canada

*Correspondence:

Paul A. Offit

offit@email.chop.edu

Specialty section:

This article was submitted to

Infectious Diseases,

a section of the journal

Frontiers in Microbiology

Received: 04 November 2019

Accepted: 19 February 2020

Published: 17 March 2020

Citation:

Geoghegan S, O'Callaghan KP and Offit PA (2020) Vaccine Safety:

Myths and Misinformation.

Front. Microbiol. 11:372.

doi: 10.3389/fmicb.2020.00372

\section{Vaccine Safety: Myths and Misinformation}

\author{
Sarah Geoghegan ${ }^{1,2}$, Kevin P. O'Callaghan' ${ }^{1}$ and Paul A. Offit ${ }^{1 *}$ \\ ${ }^{1}$ Division of Infectious Diseases, The Children's Hospital of Philadelphia, Philadelphia, PA, United States, ${ }^{2}$ National \\ Children's Research Centre, Dublin, Ireland
}

The World Health Organization has named vaccine hesitancy as one of the top ten threats to global health in 2019. The reasons why people choose not to vaccinate are complex, but lack of confidence in vaccine safety, driven by concerns about adverse events, has been identified as one of the key factors. Healthcare workers, especially those in primary care, remain key influencers on vaccine decisions. It is important, therefore, that they be supported by having easy access to trusted, evidencebased information on vaccines. Although parents and patients have a number of concerns about vaccine safety, among the most common are fears that adjuvants like aluminum, preservatives like mercury, inactivating agents like formaldehyde, manufacturing residuals like human or animal DNA fragments, and simply the sheer number of vaccines might be overwhelming, weakening or perturbing the immune system. As a consequence, some fear that vaccines are causing autism, diabetes, developmental delays, hyperactivity, and attention-deficit disorders, amongst others. In this review we will address several of these topics and highlight the robust body of scientific evidence that refutes common concerns about vaccine safety.

Keywords: vaccine, safety, adjuvant, immunization, side effect

\section{INTRODUCTION}

Vaccines are among the greatest public health achievements of modern times. They have saved and continue to save millions of lives throughout the world. Their success is such that in high income settings most patients, parents and health care providers do not have first-hand experience of the devastating consequences of many of the diseases that they prevent. Over the past few decades vaccine hesitancy has emerged as a major public health problem leading to outbreaks of communicable infections such as measles. The reasons for vaccine refusal are complex and differ according to geographical and cultural context. However, concern about vaccine safety continues to be an important driver of decreased vaccine uptake in most contexts (Larson et al., 2014). This concern is fueled by misinformation and propagated through organized antivaccine groups, social media and celebrity endorsements. Despite a wealth of scientific data supporting the safety of currently recommended vaccines, counteracting false information to convince vaccine hesitant populations continues to be challenging. While there will always be individuals with strongly held beliefs for whom well informed discussions about vaccine safety will have little impact, it has been repeatedly demonstrated that provider recommendation strongly influences decision making around vaccines (Smith et al., 2017). Thus it is important that front line health care 
workers understand the available safety data and feel empowered to give strong advice backed by scientific evidence. In this article we aim to discuss the most common vaccine safety controversies, summarize the available data, and highlight some of what we feel are the most important research studies for each topic (Table 1).

\section{DO VACCINES CAUSE AUTISM?}

The speculated link between measles, mumps, rubella vaccine (MMR) and autism spectrum disorders has a long and complicated history, beginning with a (since retracted) report in the Lancet in 1998 (Wakefield et al., 1998). The retraction occurred due to misrepresentation of clinical and biological data in the paper, issues which, in part, led to the lead author, Andrew Wakefield, being removed from the United Kingdom's medical register. This report described 12 children with a history of pervasive developmental disorder (PDD), 8 of whom had autism, along with intestinal symptomatology. A hypothesis was postulated in this paper that receipt of MMR vaccine had led to intestinal inflammation, with entrance to the bloodstream of proteins that led to brain inflammation and the development of PDD, including autism disorder. The paper was problematic in a number of ways, including that a true comparison between MMR-exposed and unexposed children was not carried out. Additionally, the only correlation between MMR-exposure and autism was report of receipt of MMR during a time period proximate to the diagnosis of autism. Since, in the natural history of the disease, the development of symptoms of autism can be expected to occur at a similar age to receipt of MMR, this apparent correlation is not surprising. Lastly, although the postulated sequence of pathogenesis had intestinal inflammation

TABLE 1 | Key concepts addressing common vaccine safety concerns.

Common vaccine Key concepts
safety myths

Too many vaccines too soon.

Too many vaccines can "overwhelm" the immune system.

MMR vaccine causes autism.

HPV vaccine increases risk of autoimmune disease.

Influenza vaccine given in early pregnancy increases risk of miscarriage.
- The number of immunologic components in vaccines have declined over time.

- The current 14 vaccines on the United States schedule contain 200 immunologic proteins in total, the smallpox vaccine contained 160.

- Epidemiologic data and biologic data show that cumulative increases in the number of vaccines have no effect on immune function.

- Original study making this claim contained 12 children, the paper was subsequently retracted due to evidence of misrepresented data.

- Multiple large scale studies, including a study of half a million children have shown no association between receipt of MMR and risk of autism.

- More than 270 million doses of HPV vaccine have been administered.

- Repeated well-designed studies show no association between HPV and Al disease.

- A study of 2762 women showed no association between influenza vaccine and spontaneous abortion. as an intermediate step, all 8 cases of autism had intestinal symptoms that started after the diagnosis of autism, not before.

Subsequent to the publication of this report, and although it was later retracted, the article led to broad media coverage of the postulated link, and widespread concern as to the safety of MMR vaccination developed. In this setting, MMR vaccine coverage in the United Kingdom and elsewhere dropped precipitously, leading to the re-emergence of measles as a public health problem and associated morbidity and mortality.

Large epidemiological studies, in particular the two referenced herein, have since clearly refuted the postulated link, and have substantively affirmed that vaccination, and the MMR vaccine in particular, have neither a correlative nor causative link to autism spectrum disorders. In 1999, 1 year after the Lancet paper, a separate retrospective cohort study of about 500 children was carried out in the North Thames region of England, examining autism spectrum disorders in children both before and after the introduction of the MMR vaccine in 1988 (Taylor et al., 1999). This study detected no differences in the rate of immunization of those children with a diagnosis of autism, as compared with those without this diagnosis. Similarly, no difference was observed in the age of diagnosis of autism between vaccinated and unvaccinated children.

A larger retrospective cohort study, and to date one of the most definitive findings of no causal link between MMR vaccine and autism, was performed by a Danish group and published in the New England Journal of Medicine in 2002 (Madsen et al., 2002). The authors reviewed the records of more than half a million children born in Denmark during the study period (19911998) and found no association between age at vaccination, receipt of vaccination, or time passed since vaccination, and the development of autism spectrum disorders.

\section{ARE TOO MANY VACCINES ON THE SCHEDULE?}

The first vaccine was introduced by Edward Jenner in 1796 and ultimately led to the worldwide eradication of smallpox (Riedel, 2005). Currently licensed vaccines are available that prevent 26 different human pathogens. Depending on geographic region children can be recommended to receive more than 30 doses of vaccines protecting as many as 20 different diseases in the first 24 months of life (World Health Organization [WHO], 2019). Children receive the most vaccines during the first year of life at a time when they are most vulnerable to the devastating consequences of invasive bacterial infection such as pneumococcal or Haemophilus influenzae meningitis. The increasing number of vaccines on the schedule has led to concerns among some vaccine hesitant groups about effects on a child's immune system and neurodevelopment. This has in turn led to the dangerous practice of individualized schedules. Timing of the recommended vaccine schedule takes into account the timing of waning of maternal antibody and maturation of the immune system, susceptibility to the disease, and effectiveness and dosing of the vaccine. Vaccines on the schedule have been tested in their final formulations and with other vaccines given 
at the same time. By addressing concerns with a non-scientific approach such as delaying or separating vaccines, providers with alternative schedules are legitimizing unfounded safety concerns and putting their patients at unnecessary risk (Offit et al., 2002; Offit and Moser, 2009).

While the number of vaccines has increased significantly over time, the numbers of immunologic components in vaccines have declined. Whereas the smallpox vaccine contained about 200 proteins, the 14 recommended vaccines on the United States schedule, combined contain about 160 immunologic components (i.e., viral proteins, bacterial proteins, and bacterial polysaccharides) (Offit et al., 2002). Three factors account for this decline: first, the worldwide eradication of smallpox obviated the need for that vaccine; second, advances in protein chemistry and protein purification; and third, the birth of recombinant DNA technology have resulted in vaccines containing fewer antigens.

\section{CAN RECEIVING MULTIPLE VACCINES WEAKEN THE IMMUNE SYSTEM?}

Epidemiologic and immunologic data refute the concept that vaccines dampen the immune response. In a large Danish cohort study comprising 805,206 children born between 1990 and 2001 no adverse associations were observed between an increasing number of vaccinations and hospitalizations with non-vaccine-targeted infections (Hviid et al., 2005). A second nested case control study included 944 patients from 6 United States healthcare organizations to examine cumulative vaccine antigen exposure in the first 24 months of life and risk of non-vaccine-targeted infections from 24-47 months. The group found no differences in cumulative vaccine antigen exposure between children who were admitted to hospital with a non-vaccine targeted infection and those who were not (Glanz et al., 2018). The epidemiologic data is supported by biological evidence. A study in Canada investigating the impact of vaccination on immune status compared the immune response to general non-antigen-specific stimuli in entirely unvaccinated versus vaccinated children at 3-5 years of age. Innate and adaptive responses were compared. Investigators did not find any differences in immunological outcomes in vaccinated children. Furthermore, and notably, equivalently robust innate and adaptive responses to pathogen associated microbial patterns and generic T-cell stimulants were seen in both groups (Sherrid et al., 2017).

\section{DOES GIVING VACCINES IN THE FIRST 24 MONTHS OF LIFE IMPACT NEURODEVELOPMENTAL OUTCOME?}

Facing the large body of safety data demonstrating no link between MMR vaccine and autism, a new concern frequently cited by antivaccine groups is that the number of antigens children are exposed to in the first year of life has an adverse effect on neurodevelopment. This has also been well studied and there has been no correlation found between the number of vaccine antigens received and adverse neuropsychological outcomes (Iqbal et al., 2013), nor has a difference been demonstrated in neuropsychological outcomes in those who received vaccines on time in the first year of life compared to delayed schedules (Smith and Woods, 2010).

\section{ARE THE ADJUVANTS AND PRESERVATIVES USED IN VACCINES SAFE?}

Since large-scale epidemiological studies have broadly disproven any link between the vaccine antigens and development of adverse neurodevelopmental outcomes, more recent vaccine hesitancy efforts have centered around concerns as to the safety of a variety of vaccine ingredients - including adjuvants and preservatives. Two of the more commonly cited of these are aluminum and mercury.

Aluminum is the third most abundant element in the environment, and the most abundant metal, and is found diffusely in soil, water, plants, and air. It is found in a variety of consumer products, as well as throughout the human food chain, and in many pharmaceuticals. In vaccines, aluminum is used as an adjuvant, a component that boosts immune response to the vaccine antigens. When adjuvants are used, they allow for smaller amounts of vaccine to be given, as well as fewer doses. Aluminum has been used in a variety of vaccines, including hepatitis A and $\mathrm{B}, H$. influenzae type $\mathrm{b}$, and pneumococcal vaccines.

As a vaccine component, aluminum has been extensively tested for safety as part of pre-licensure clinical trials. We know from studies examining the aluminum exposure of infants that the cumulative amount of aluminum from vaccines in the first 6 months of life is actually far less than that received from dietary sources, including both breast milk and formula (Keith et al., 2002; Mitkus et al., 2011). Both sources represent far less exposure than that represented by a regulatory minimal risk level (MRL), which is established by the Agency for Toxic Substances and Disease Registry. The safety of aluminum salts from vaccines, and from other sources, has also been established in studies examining blood and hair levels of aluminum in infants, and associated neurodevelopmental outcomes (Karwowski et al., 2018). Such studies have not proven a link between blood and hair concentrations of aluminum and receipt of vaccines, nor between blood and hair aluminum concentrations and neurodevelopmental outcomes.

Mercury, or more specifically a compound containing ethylmercury called thimerosal, has been used at various points in the past as a preservative in multidose vials of vaccine. Because some forms of mercury are known to be neurotoxic, this has led to concerns in the past that this mercury-containing compound could represent a danger to patient safety. However, the form of mercury contained in thimerosal (ethylmercury) is substantially different from the more toxic methylmercury, despite the similarity in their names. Ethylmercury is cleared quickly from human tissues, and does not accumulate substantially, unlike methylmercury. When the safety of thimerosal as a vaccine 
component has been examined, it has not been found to have any associated risks, including no evidence of an increased risk of autism (Tozzi et al., 2009; Price et al., 2010).

\section{DO VACCINES CONTAIN HUMAN AND ANIMAL DNA FRAGMENTS?}

Due to the fact that some vaccines are manufactured using human embryo cell lines, some residual human DNA can be found in a variety of vaccines, including varicella, rubella, hepatitis $A$, and one of the rabies vaccines. The exposure of vaccinated patients to such DNA has been raised as a potential safety concern. There are a number of reasons to believe that this exposure does not present a danger to vaccine recipients.

Primary amongst these reasons is the fact that human DNA is highly sensitive to destruction by chemical processes, and much of the DNA involved in creating these vaccines is destroyed in the process. The end result contains only minimal amounts of residual DNA, all of it fragmented, and none of it representing a viable genome. Additionally, isolated portions of DNA cannot incorporate themselves into a new genome without many additional processes being involved. In fact, this involves many of the major issues that make gene therapy difficult.

The safety of tiny amounts of residual human DNA has been assessed by at least two investigator groups, who have used both probabilistic modeling and animal models. These studies all generally concur that dose equivalents on the order of millions to trillions of the amount of DNA contained in vaccines would be required before risk of, for example, an oncogenic event, would become appreciable (Wierenga et al., 1995; Yang, 2013).

\section{DO VACCINES CAUSE AUTOIMMUNE DISEASES?}

Associations between vaccines and autoimmunity are another frequently cited safety concern and have been heavily studied in relation to a number of different autoimmune (AI) disorders including diabetes mellitus type 1 (DM type 1), Guillain Barré Syndrome (GBS), multiple sclerosis (MS) and other demyelinating disorders. Existing epidemiologic studies have found no associations between the number of different vaccines and an increased risk of autoimmune disorders. Two recent systematic reviews looking at the association between different individual vaccines and central demyelinating disorders (hepatitis B, human papilloma virus (HPV), influenza, MMR, varicella, tetanus, Bacillus Calmette Guérin (BCG), polio or diphtheria) concluded that there was no relationship between receipt of a vaccine and development of MS (Mailand and Frederiksen, 2017; Mouchet et al., 2018). Links with DM type 1 have also been extensively studied. A German study which included more than 1900 children from a prospective cohort data set found no association between vaccination and development of DM type 1 in children with a high familial risk of AI disorders (Beyerlein et al., 2017). A metanalysis of 23 different case control studies including 11 different vaccines and 13,000 patients found no association between vaccination and risk of DM type 1 (Morgan et al., 2016).

The Advisory Committee on Immunization Practices (ACIP) give a precautionary recommendation on influenza vaccine for patients with a history of Guillain Barré Syndrome within 6 weeks of receiving the vaccine due to data suggesting a possible marginal increased risk. The data on this association is variable and has been detected in some seasons but not in others. A metanalysis of 39 controlled observational studies found that the relative risk of GBS following receipt of any influenza vaccine was 1.41 (Martin Arias et al., 2015). The Vaccine Safety Datalink team, in a self-controlled risk interval analysis of data from the 2009-2010 influenza season found an association between risk of GBS and the monovalent inactivated vaccine (MIV) against H1N1 but not in association with the trivalent inactivated vaccine (TIV) (Greene et al., 2012). However, in a later analysis that included the 20102011 season and adjusted for antecedent respiratory infection there was no increased risk following 1.27 million 200910 MIV or 2.8 million 2010-11 TIV doses (Greene et al., 2013). The risk of GBS within 6 weeks of influenza infection is greater than after the vaccine. A Canadian study which used a self-controlled risk interval design looked at 2831 admissions for Guillain-Barré syndrome; 330 received an influenza vaccine and 109 had an influenza-coded healthcare encounter within 42 weeks before hospitalization. The attributable risks of admissions for GBS within 6 weeks were 1.03 per million vaccinations, compared with 17.2 GBS admissions per million influenza-coded health-care encounters (Kwong et al., 2013).

In recent years, the HPV vaccine has been a particular subject of concern. The misinformation surrounding HPV vaccines has tragically resulted in lower uptake in many countries, denying millions of adolescents the opportunity to be protected from fatal HPV-related cancers in later life. Meanwhile in Australia, where high uptake has been maintained, a recent modeling study indicates progress toward eliminating cervical cancer as a public health problem (Hall et al., 2019). Since entering the market in 2006 more than 270 million doses of the HPV vaccine have been administered worldwide, including 100 million in the United States (Centers for Disease Control and Prevention [CDC], 2019). Studies have repeatedly demonstrated that this is a safe vaccine and there are strong epidemiologic data refuting claims of an association with AI diseases. These include (most prominently) a large population-based cohort in Denmark and Sweden that analyzed more than 696,000 doses of HPV-4 vaccine and found no evidence of an association with AI disorders (Arnheim-Dahlstrom et al., 2013). Additionally, a large case control study in France matched 211 definite cases of AI disease with 875 controlls. No association was found between HPV vaccine an several AI disorders (i.e., immune thrombocytopenic purpura [ITP], GBS, connective tissue disorders, DM type 1, and autoimmune thyroiditis) (Grimaldi-Bensouda et al., 2017). This study is consistent with the findings of a registry based cohort study in Finland which included 134,615 vaccinated females and (Skufca et al., 2018) a review of 6 years of post-licensure data in the Vaccine Adverse Event Reporting 
System (VAERS) which identified no unexpected safety concerns (Arana et al., 2018).

\section{ARE THE VACCINES CURRENTLY GIVEN IN PREGNANCY SAFE?}

While vaccines have significantly contributed to a reduction in child mortality, progress in reducing deaths in infants that are too young to receive vaccines has been slower. Administration of vaccines to expectant mothers has the potential to protect the most vulnerable infants from serious illness and death as well as protecting women themselves from increased morbidity and mortality during a time of altered immune function. Influenza and pertussis vaccines (Tdap) are recommended to be given during pregnancy in most high-income countries. Tetanus vaccine remains a recommendation in settings where there is an ongoing risk of neonatal tetanus. Recent data from the CDC indicate that from 2010-2018 pregnant women accounted for 24$34 \%$ of influenza-associated hospitalizations per season among women aged 15-44 years and a total of 3,928 pertussis-related hospitalizations among infants aged $<2$ months (Lindley et al., 2019). Despite this clear burden of disease vaccination rates during pregnancy remain poor. Influenza and Tdap vaccination coverage rates during pregnancy in the United States reported in April 2019 were 53.7 and $54.9 \%$, respectively (Lindley et al., 2019). Safety concerns and provider recommendation are among the most important factors influencing vaccine uptake in pregnancy (Wilson et al., 2015).

Influenza and pertussis vaccines during pregnancy are recommended by both federal agencies and non-governmental advisory groups. Because millions of pregnant women have received the influenza vaccine during pregnancy there is a large body of data confirming that it is safe. Numerous well-designed cohort studies have found no association between influenza vaccine and adverse pregnancy and infant outcomes including birth malformations, still birth, spontaneous abortion, low birth weight and low Apgar scores (Kharbanda et al., 2013, 2017; Zerbo et al., 2017). Impact of timing of vaccination including risk of administration in the first trimester has also been studied and repeatedly shown to be safe (Kharbanda et al., 2017). A critical review of a 2017 study that claimed a theoretical association between influenza vaccine and spontaneous abortion identified a number of methodological flaws including small sample size and in appropriate matching of cases and controls. A follow up study by the same group looking at a larger sample size of 2762 women (three times the amount of the original paper) showed no significant association between spontaneous abortion and influenza vaccine regardless of prior season vaccination status (Donahue et al., 2019). Similarly pertussis vaccine has been shown to be safe in pregnancy in numerous well designed cohort studies (Sukumaran et al., 2015; DeSilva et al., 2016, 2017; Layton et al., 2017). The greatest challenge facing vaccination uptake in pregnancy is not a lack of available safety data but a failure to communicate this adequately to patients. There are currently a number of vaccines in development that are intended to be given to pregnant women including vaccines protecting against respiratory syncytial virus, group B streptococcus, Zika virus and CMV. Given this landscape it is crucial that there is a focused effort to improve confidence in vaccine safety during pregnancy among both pregnant women and providers (Heath et al., 2017).

\section{DO VACCINES HAVE ANY PROVEN SEVERE OR LIFE-THREATENING SIDE EFFECTS?}

Vaccines are given to healthy people and thus are held to an even higher safety standard than medicines used to treat diseases. They undergo rigorous safety and efficacy studies in the prelicensure stage. In order to identify rare side effects that may have been missed in pre-licensure studies vaccines undergo ongoing post-licensure monitoring. The infrastructure in place provides robust data about rare side effects of vaccines. Anaphylaxis is example of an established rare but life threatening side effect of vaccines with the most recent United States data from the vaccine safety data link identifying a rate of anaphylaxis of 1.31 (95\% CI, 0.90-1.84) per million vaccine doses and no deaths (McNeil et al., 2016).

When considering very rare side effects of vaccines a balance should be sought between the risk of a side effect occurring and the risk of disease occurring if the vaccine is not given. An illustrative example is the use of the oral polio vaccine (OPV). Vaccine strain-related paralytic polio will occur in about 1 in 2.5 million people who receive this vaccine. The oral polio vaccine was used in the United States for 40 years, leading to eradication of the disease in 1979. Since the year 2000 the inactivated polio vaccine (IPV) has been the only polio vaccine recommended for use in the United States (Centers for Disease Control and Prevention [CDC], 2019). This decision was made because the potential, however, rare, for paralysis due to OPV, was considered unacceptable when a feasible alternative existed. However, in many low-income settings, IPV was not a feasible alternative and thus the risk equation was different. OPV is cheaper and more convenient to administer and has led to the near global eradication of disease. The number of cases of wild type polio worldwide has decreased by $99 \%$ from 1988 to 2018, from an estimated $350000+$ cases (1988) to 33 reported cases (2018) (World Health Organization [WHO], 2019). Complete eradication worldwide will ultimately require the cessation of use of trivalent OPV, a strategy currently being implemented by the WHO through a synchronized switch to a bivalent oral polio vaccine, successfully implemented in 150 countries as of 2016 (Hampton et al., 2016). Currently, poliovirus types 2 and 3 have been eliminated from the world.

\section{CONCLUSION}

In this article we summarize common vaccine safety controversies and highlight what we feel are some of the most important studies refuting these concerns. The misinformation 
surrounding vaccine safety poses a threat to children's lives worldwide and it is crucial that primary care workers at the front line understand the evidence and can confidently communicate the message that vaccines are a safe and lifesaving intervention.

\section{REFERENCES}

Arana, J. E., Harrington, T., Cano, M., Lewis, P., Mba-Jonas, A., Rongxia, L., et al. (2018). Post-licensure safety monitoring of quadrivalent human papillomavirus vaccine in the Vaccine Adverse Event Reporting System (VAERS), 2009-2015. Vaccine 36, 1781-1788. doi: 10.1016/j.vaccine.2018.02.034

Arnheim-Dahlstrom, L., Pasternak, B., Svanstrom, H., Sparen, P., and Hviid, A. (2013). Autoimmune, neurological, and venous thromboembolic adverse events after immunisation of adolescent girls with quadrivalent human papillomavirus vaccine in Denmark and Sweden: cohort study. BMJ 347:f5906. doi: 10.1136/bmj.f5906

Beyerlein, A., Strobl, A. N., Winkler, C., Carpus, M., Knopff, A., Donnachie, E., et al. (2017). Vaccinations in early life are not associated with development of islet autoimmunity in type 1 diabetes high-risk children: results from prospective cohort data. Vaccine 35, 1735-1741. doi: 10.1016/j.vaccine.2017. 02.049

Centers for Disease Control and Prevention [CDC] (2019). HPV Vaccination is Safe and Effective. Available online at: https://www.cdc.gov/hpv/parents/ vaccinesafety.html (accessed October 30, 2019).

DeSilva, M., Vazquez-Benitez, G., Nordin, J. D., Lipkind, H. S., Klein, N. P., Cheetham, T. C., et al. (2017). Maternal Tdap vaccination and risk of infant morbidity. Vaccine 35, 3655-3660. doi: 10.1016/j.vaccine.2017.05.041

DeSilva, M., Vazquez-Benitez, G., Nordin, J. D., Lipkind, H. S., Romitti, P. A., DeStefano, F., et al. (2016). Tdap vaccination during pregnancy and microcephaly and other structural birth defects in offspring. JAMA 316, 18231825.

Donahue, J. G., Kieke, B. A., King, J. P., Mascola, M. A., Shimabukuro, T. T., DeStefano, F., et al. (2019). Inactivated influenza vaccine and spontaneous abortion in the Vaccine Safety Datalink in 2012-13, 2013-14, and 2014-15. Vaccine 37, 6673-6681. doi: 10.1016/j.vaccine.2019.09.035

Glanz, J. M., Newcomer, S. R., Daley, M. F., DeStefano, F., Groom, H. C., Jackson, M. L., et al. (2018). Association between estimated cumulative vaccine antigen exposure through the first 23 months of life and non-vaccine-targeted infections from 24 through 47 months of age. JAMA 319, 906-913. doi: 10.1001/jama. 2018.0708

Greene, S. K., Rett, M., Weintraub, E. S., Li, L., Yin, R., Amato, A. A., et al. (2012). Risk of confirmed Guillain-Barre syndrome following receipt of monovalent inactivated influenza A (H1N1) and seasonal influenza vaccines in the Vaccine Safety Datalink Project, 2009-2010. Am. J. Epidemiol. 175, 1100-1109. doi: 10.1093/aje/kws195

Greene, S. K., Rett, M. D., Vellozzi, C., Li, L., Kulldorff, M., Marcy, S. M., et al. (2013). Guillain-Barre Syndrome, influenza vaccination, and antecedent respiratory and gastrointestinal infections: a case-centered analysis in the Vaccine Safety Datalink, 2009-2011. PLoS One 8:e67185. doi: 10.1371/journal. pone.0067185

Grimaldi-Bensouda, L., Rossignol, M., Kone-Paut, I., Krivitzky, A., Lebrun-Frenay, C., Clet, J., et al. (2017). Risk of autoimmune diseases and human papilloma virus (HPV) vaccines: six years of case-referent surveillance. J. Autoimmun. 79, 84-90. doi: 10.1016/j.jaut.2017.01.005

Hall, M. T., Simms, K. T., Lew, J. B., Smith, M. A., Brotherton, J. M., Saville, M., et al. (2019). The projected timeframe until cervical cancer elimination in Australia: a modelling study. Lancet Public Health 4, e19-e27. doi: 10.1016/S2468-2667(18) 30183-X

Hampton, L. M., Farrell, M., Ramirez-Gonzalez, A., Menning, L., Shendale, S., Lewis, I., et al. (2016). Cessation of trivalent oral poliovirus vaccine and introduction of inactivated poliovirus vaccine - worldwide. MMWR Morb. Mortal. Wkly. Rep. 2016, 934-938.

Heath, P. T., Culley, F. J., Jones, C. E., Kampmann, B., Le Doare, K., Nunes, M. C., et al. (2017). Group B Streptococcus and respiratory syncytial virus immunisation during pregnancy: a landscape analysis. Lancet Infect. Dis. 17, e223-e234. doi: 10.1016/S1473-3099(17)30232-3

\section{AUTHOR CONTRIBUTIONS}

SG and $\mathrm{KO}^{\prime} \mathrm{C}$ reviewed the literature and wrote the manuscript. $\mathrm{PO}$ designed, reviewed, and edited the manuscript.

Hviid, A., Wohlfahrt, J., Stellfeld, M., and Melbye, M. (2005). Childhood vaccination and nontargeted infectious disease hospitalization. JAMA 294, 699-705.

Iqbal, S., Barile, J. P., Thompson, W. W., and DeStefano, F. (2013). Number of antigens in early childhood vaccines and neuropsychological outcomes at age 7-10 years. Pharmacoepidemiol. Drug Saf. 22, 1263-1270. doi: 10.1002/pds. 3482

Karwowski, M. P., Stamoulis, C., Wenren, L. M., Faboyede, G. M., Quinn, N., Gura, K. M., et al. (2018). Blood and hair aluminum levels, vaccine history, and early infant development: a cross-sectional study. Acad. Pediatr. 18, 161-165. doi: 10.1016/j.acap.2017.09.003

Keith, L. S., Jones, D. E., and Chou, C. H. (2002). Aluminum toxicokinetics regarding infant diet and vaccinations. Vaccine 20(Suppl. 3), S13-S17.

Kharbanda, E. O., Vazquez-Benitez, G., Lipkind, H., Naleway, A., Lee, G., and Nordin, J. D. (2013). Inactivated influenza vaccine during pregnancy and risks for adverse obstetric events. Obstet. Gynecol. 122, 659-667. doi: 10.1097/AOG. 0b013e3182a1118a

Kharbanda, E. O., Vazquez-Benitez, G., Romitti, P. A., Naleway, A. L., Cheetham, T. C., Lipkind, H. S., et al. (2017). First trimester influenza vaccination and risks for major structural birth defects in offspring. J. Pediatr. 187, 234-239.e4. doi: 10.1016/j.jpeds.2017.04.039

Kwong, J. C., Vasa, P. P., Campitelli, M. A., Hawken, S., Wilson, K., Rosella, L. C., et al. (2013). Risk of Guillain-Barre syndrome after seasonal influenza vaccination and influenza health-care encounters: a self-controlled study. Lancet Infect. Dis. 13, 769-776. doi: 10.1016/S1473-3099(13)70104-X

Larson, H. J., Jarrett, C., Eckersberger, E., Smith, D. M., and Paterson, P. (2014). Understanding vaccine hesitancy around vaccines and vaccination from a global perspective: a systematic review of published literature, 2007-2012. Vaccine 32, 2150-2159. doi: 10.1016/j.vaccine.2014.01.081

Layton, J. B., Butler, A. M., Li, D., Boggess, K. A., Weber, D. J., McGrath, L. J., et al. (2017). Prenatal Tdap immunization and risk of maternal and newborn adverse events. Vaccine 35, 4072-4078. doi: 10.1016/j.vaccine.2017.06.071

Lindley, M. C., Kahn, K. E., Bardenheier, B. H., D’Angelo, D. V., Dawood, F. S., Fink, R. V., et al. (2019). Vital signs: burden and prevention of influenza and pertussis among pregnant women and infants - United States. MMWR Morb. Mortal. Wkly. Rep. 68, 885-892. doi: 10.15585/mmwr.mm6840e1

Madsen, K. M., Hviid, A., Vestergaard, M., Schendel, D., Wohlfahrt, J., Thorsen, P., et al. (2002). A population-based study of measles, mumps, and rubella vaccination and autism. N. Engl. J. Med. 347, 1477-1482.

Mailand, M. T., and Frederiksen, J. L. (2017). Vaccines and multiple sclerosis: a systematic review. J. Neurol. 264, 1035-1050. doi: 10.1007/s00415-016-8263-4

Martin Arias, L. H., Sanz, R., Sainz, M., Treceno, C., and Carvajal, A. (2015). Guillain-Barre syndrome and influenza vaccines: a meta-analysis. Vaccine 33, 3773-3778. doi: 10.1016/j.vaccine.2015.05.013

McNeil, M. M., Weintraub, E. S., Duffy, J., Sukumaran, L., Jacobsen, S. J., Klein, N. P., et al. (2016). Risk of anaphylaxis after vaccination in children and adults. J. Allergy Clin. Immunol. 137, 868-878. doi: 10.1016/j.jaci.2015.07.048

Mitkus, R. J., King, D. B., Hess, M. A., Forshee, R. A., and Walderhaug, M. O. (2011). Updated aluminum pharmacokinetics following infant exposures through diet and vaccination. Vaccine 29, 9538-9543. doi: 10.1016/j.vaccine. 2011.09.124

Morgan, E., Halliday, S. R., Campbell, G. R., Cardwell, C. R., and Patterson, C. C. (2016). Vaccinations and childhood type 1 diabetes mellitus: a meta-analysis of observational studies. Diabetologia 59, 237-243. doi: 10.1007/s00125-0153800-8

Mouchet, J., Salvo, F., Raschi, E., Poluzzi, E., Antonazzo, I. C., De Ponti, F., et al. (2018). Hepatitis B vaccination and the putative risk of central demyelinating diseases - a systematic review and meta-analysis. Vaccine 36, 1548-1555. doi: 10.1016/j.vaccine.2018.02.036

Offit, P. A., and Moser, C. A. (2009). The problem with Dr Bob's alternative vaccine schedule. Pediatrics 123, e164-e169. doi: 10.1542/peds.2008-2189 
Offit, P. A., Quarles, J., Gerber, M. A., Hackett, C. J., Marcuse, E. K., Kollman, T. R., et al. (2002). Addressing parents' concerns: do multiple vaccines overwhelm or weaken the infant's immune system? Pediatrics 109, 124-129. doi: 10.1542/ peds.109.1.124

Price, C. S., Thompson, W. W., Goodson, B., Weintraub, E. S., Croen, L. A., Hinrichsen, V. L., et al. (2010). Prenatal and infant exposure to thimerosal from vaccines and immunoglobulins and risk of autism. Pediatrics 126, 656-664. doi: 10.1542/peds.2010-0309

Riedel, S. (2005). Edward Jenner and the history of smallpox and vaccination. Proc. Bayl. Univ. Med. Cent. 18, 21-25. doi: 10.1080/08998280.2005.11928028

Sherrid, A. M., Ruck, C. E., Sutherland, D., Cai, B., and Kollmann, T. R. (2017). Lack of broad functional differences in immunity in fully vaccinated vs. unvaccinated children. Pediatr. Res. 81, 601-608. doi: 10.1038/pr.2016.272

Skufca, J., Ollgren, J., Artama, M., Ruokokoski, E., Nohynek, H., and Palmu, A. A. (2018). The association of adverse events with bivalent human papilloma virus vaccination: a nationwide register-based cohort study in Finland. Vaccine 36, 5926-5933. doi: 10.1016/j.vaccine.2018.06.074

Smith, L. E., Amlot, R., Weinman, J., Yiend, J., and Rubin, G. J. (2017). A systematic review of factors affecting vaccine uptake in young children. Vaccine 35, 6059-6069. doi: 10.1016/j.vaccine.2017.09.046

Smith, M. J., and Woods, C. R. (2010). On-time vaccine receipt in the first year does not adversely affect neuropsychological outcomes. Pediatrics 125, 1134-1141. doi: 10.1542/peds.2009-2489

Sukumaran, L., McCarthy, N. L., Kharbanda, E. O., McNeil, M. M., Naleway, A. L., Klein, N. P., et al. (2015). Association of Tdap vaccination with acute events and adverse birth outcomes among pregnant women with prior tetanus-containing immunizations. JAMA 314, 1581-1587. doi: 10.1001/jama.2015.12790

Taylor, B., Miller, E., Farrington, C. P., Petropoulos, M. C., Favot-Mayaud, I., Li, J., et al. (1999). Autism and measles, mumps, and rubella vaccine: no epidemiological evidence for a causal association. Lancet 353, 2026-2029.

Tozzi, A. E., Bisiacchi, P., Tarantino, V., De Mei, B., D’Elia, L., Chiarotti, F., et al. (2009). Neuropsychological performance 10 years after immunization in infancy with thimerosal-containing vaccines. Pediatrics $123,475-482$. doi: 10.1542/peds.2008-0795

Wakefield, A. J., Murch, S. H., Anthony, A., Linnell, J., Casson, D. M., Malik, M., et al. (1998). Ileal-lymphoid-nodular hyperplasia, non-specific colitis, and pervasive developmental disorder in children. Lancet 351, 637-641. doi: 10. 1016/s0140-6736(97)11096-0

Wierenga, D. E., Cogan, J., and Petricciani, J. C. (1995). Administration of tumor cell chromatin to immunosuppressed and non-immunosuppressed non-human primates. Biologicals 23, 221-224. doi: 10.1006/biol.1995.0036

Wilson, R. J., Paterson, P., Jarrett, C., and Larson, H. J. (2015). Understanding factors influencing vaccination acceptance during pregnancy globally: a literature review. Vaccine 33, 6420-6429. doi: 10.1016/j.vaccine.2015.08.046

World Health Organization [WHO] (2019). Immunization, Vaccines and Biologicals: WHO Recommendations for Routine Immunization - Summary Tables. Available online at: https:/www.who.int/immunization/policy/ immunization_tables/en/ (accessed October 30, 2019).

Yang, H. (2013). Establishing acceptable limits of residual DNA. PDA J. Pharm. Sci. Technol. 67, 155-163. doi: 10.5731/pdajpst.2013.00910

Zerbo, O., Modaressi, S., Chan, B., Goddard, K., Lewis, N., Bok, K., et al. (2017). No association between influenza vaccination during pregnancy and adverse birth outcomes. Vaccine 35, 3186-3190. doi: 10.1016/j.vaccine.2017.04.074

Conflict of Interest: The authors declare that the research was conducted in the absence of any commercial or financial relationships that could be construed as a potential conflict of interest.

Copyright (C) 2020 Geoghegan, O'Callaghan and Offit. This is an open-access article distributed under the terms of the Creative Commons Attribution License (CC BY). The use, distribution or reproduction in other forums is permitted, provided the original author(s) and the copyright owner(s) are credited and that the original publication in this journal is cited, in accordance with accepted academic practice. No use, distribution or reproduction is permitted which does not comply with these terms. 